\title{
Distributed Event-Triggered Control for Synchronization of Dynamical Networks with Estimators *
}

\author{
Tao Liu* Ming Cao* Claudio De Persis* \\ Julien M. Hendrickx ${ }^{* *}$ \\ * Faculty of Mathematics and Natural Sciences, \\ University of Groningen, 9747 AG, Groningen, The Netherlands \\ (e-mail: t.liu@rug.nl,m.cao@rug.nland c.de.persis@rug.nl) \\ ** ICTEAM Institute, The Université catholique de Louvain, \\ Louvain-la-Neuve, Belgium \\ (e-mail: julien.hendrickx@uclouvain.be)
}

\begin{abstract}
This paper studies the synchronization problem of a dynamical network with eventbased communication, where each node communicates to its neighbors only when an eventtriggering condition is fulfilled. In order to achieve asymptotic synchronization as well as to prevent the occurrence of Zeno behavior, estimators are introduced into each node to estimate the current state of its neighbors and of its own. Then, with the assistance of these estimators, a distributed event-triggering rule is designed, which only depends on the information that the node can obtain, and thus can be implemented in a decentralized way. Finally, a numerical example is given to show the effectiveness of the proposed results.
\end{abstract}

Keywords: Dynamical networks, synchronization, distributed event-triggered control.

\section{INTRODUCTION}

Nowadays, more and more control systems are implemented through communication networks. In order to use the limited communication network resources effectively, event-triggered control has been introduced in the field of networked control system (NCS) and has received attention increasingly in recent years (Heemels et al. (2012)).

By sampling the outputs based on the occurrence of a well-defined event, a system with an event-triggered controller can adjust its sample rates adaptively according to what is happening within the controlled system, and hence can reduce unnecessary communications effectively. In Tabuada (2007) and Mazo Jr. and Cao (2011), the stabilization problem of nonlinear NCSs was investigated by utilizing centralized and decentralized event-trigged control methods, respectively, in the framework of inputto-state stability of nonlinear systems. Output decentralized event-triggered control for a linear NCS was studied via stability theory of impulsive systems in Donkers and Heemels (2012). To enlarge the inter-execution intervals,

\footnotetext{
* The work of Liu and Cao was supported by the EU INTERREG program under the auspices of the SMARTBOT project. The work of Cao was also supported by the European Research Council (ERC-StG-307207). The work of De Persis was partially supported by the Dutch Organization for Scientific Research (NWO) under the auspices of the project Quantized Information Control for formation Keeping (QUICK). The work of Hendrickx was supported by the Belgian Network DYSCO (Dynamical Systems, Control, and Optimization), funded by the Interuniversity Attraction Poles Program, initiated by the Belgian Science Policy Office. His work was also supported by the Concerted Research Action (ARC) Large Graphs and Networks of the French Community of Belgium.
}

model-based event-triggered control was proposed also for a linear NCS in Garcia and Antsaklis (2013). In addition, distributed event-triggered control for a system with interconnected subsystems was developed in Wang and Lemmon (2011) and De Persis et al. (2011) by using the small gain theory for large-scale systems.

Motivated by the fact that connected nodes in some realworld networks share information over a digital platform, the problem of synchronization of dynamical networks and its related topic - consensus of multi-agent systems have also been investigated under the circumstance that nodes or agents communicate with each other only at some discrete time instances that are based on the occurrence of a well-defined event. In Dimarogonas and Johansson (2009), a distributed event-triggered control mechanism was developed to investigate asymptotic consensus of a multiagent system. This control method was further extended to studying $L_{2}$ gain stability of the system with additive disturbances in Dimarogonas (2011). To guarantee asymptotic consensus as well as prevent the occurrence of Zeno behavior, a threshold exponentially decreasing in time was introduced into a decentralized event-triggering rule in Seyboth et al. (2013). However, these works only focused on dynamical networks or multi-agent systems with simple node dynamics (single-integrators or double-integrators), and do not appear to extend in a straightforward way to networks with more general node dynamics.

Most recently, for a network with generalized linear node dynamics, a new distributed event-triggered control method was introduced in Liu et al. (2012), under which asymptotic synchronization of the network can be 
achieved. But there is no evidence that the designed eventtriggering rule can prevent Zeno behaviors. Moreover, in Demir and Lunze (2012), estimators were introduced into each node, and were used to design a decentralized eventtriggering rule with a fixed threshold, but only bounded synchronization was obtained.

In this paper, we will study asymptotic synchronization of a dynamical network with both event-based communication and generalized linear node dynamics by designing a distributed event-triggering rule. It is known that the Zeno behavior, in which two consecutive execution times approach arbitrarily closely resulting in an accumulation point is undesirable in practice (Tabuada (2007)). Thus, the key problem is to design a proper event-triggering rule such that the network can achieve asymptotic synchronization without Zeno behaviors. Moreover, each node in such a network can only get information from its neighbors and also only at some discrete time instances, therefore, it is more practical to design an event-triggering rule by only using these limited information. This in turn increases the difficulty of the design problem. To overcome these obstacles, we introduce estimators into each node. The estimators built in each node provides the node with estimations of its neighbors by using the limited information that the node can obtain. Then with the help of these estimators, we design a distributed event-triggering rule such that the problem can be solved.

Notation: We denote by $\mathbb{R}, \mathbb{R}^{+}$and $\mathbb{Z}^{+}$the set of real numbers, non-negative numbers and non-negative integers, respectively; by $\mathbb{R}^{n}$ and $\mathbb{R}^{n \times m}$ the set of $n$-dimensional real vector and $n \times m$ real matrix. $I_{n}, 1_{n}$ and $1_{n \times m}$ are the $n$-dimensional identity matrix, the $n$-dimensional vector with all entries being 1 and the $n \times m$ matrix also with all entries being 1 , respectively. We use $\|\cdot\|$ to represent the Euclidean norm of a vector $x \in \mathbb{R}^{n}$ and also the induced norm of a matrix $A \in \mathbb{R}^{n \times m}$. "T" is the transpose of a vector or a matrix, " $-1 "$ is the inverse of a nonsingular matrix, and " $\otimes$ " is the Kronecker product of two matrices. $\lambda(\cdot)$ denotes all the eigenvalues of a square matrix, $\operatorname{Re}(\cdot)$ represents the real part of a complex number.

\section{NETWORK MODEL AND PRELIMINARIES}

Consider a network of dynamical systems interconnected via a digital platform. The state equations of the network are given as follows

$$
\dot{x}_{i}(t)=H x_{i}(t)+\Gamma u_{i}, \quad i=1,2, \ldots, N,
$$

where $x_{i}=\left(x_{i 1}, x_{i 2}, \ldots, x_{i n}\right)^{\top} \in \mathbb{R}^{n}$ is the state of node i. $H \in \mathbb{R}^{n \times n}$ is a constant matrix describing the node dynamics. $\Gamma \in \mathbb{R}^{n \times n}$ is the inner coupling matrix. $u_{i} \in \mathbb{R}^{n}$ is the control input of node $i$. We suppose that the topological structure of the network is given and is represented by an outer coupling matrix $A=\left(a_{i j}\right)_{N \times N} \in \mathbb{R}^{N \times N}$. Here, we are only interested in undirected networks, i.e., if there is a connection between nodes $i$ and $j(i \neq j)$, then $a_{i j}=a_{j i}=1$; otherwise $a_{i j}=a_{j i}=0$. The diagonal entries of $A$ satisfy

$$
a_{i i}=-\sum_{j \in \mathcal{N}_{i}} a_{i j}=-\sum_{j \in \mathcal{N}_{i}} a_{j i}, \quad i=1,2, \ldots, N .
$$

The problem to be considered is with the given network topology, how to design the control input $u_{i}$ and mean- while how to determine the time sequence $\left\{t_{k_{i}}^{i}\right\}, k_{i} \in \mathbb{Z}^{+}$ when node $i$ should communicate to its neighbors (i.e., samples its state and then sends the sampled value to its neighbors) such that network (1) can achieve synchronization asymptotically.

To reduce the unnecessary communication between interconnected nodes as well as to achieve asymptotic synchronization, we will adopt event-triggered control, i.e., design an event-triggering rule to determine such a time sequence $\left\{t_{k_{i}}^{i}\right\}$. In this case, Zeno behavior which is undesirable in practice may occur. So it is important to exclude the occurrence of such a behavior by designing a proper control input $u_{i}$ and a well-defined event-triggering rule for each node, and that is the main purpose of this paper.

We assume that the network is connected via a communication network, and each node in the network can only access values of its neighbors at certain discrete time instances. It is likely that these limited information is insufficient for the design purpose, in particular for networks whose nodes will synchronize to a time-varying trajectory. To solve this problem, we adopt control input $u_{i}$ as follows

$$
u_{i}=c \sum_{j \in \mathcal{N}_{i}} a_{i j}\left(\hat{x}_{j}^{i}(t)-\hat{x}_{i}^{i}(t)\right), i=1,2, \ldots N,
$$

where $c>0$ is the coupling strength, and $a_{i j}$ are entries of matrix $A . \hat{x}_{j}^{i}=\left(\hat{x}_{j 1}^{i}, \hat{x}_{j 2}^{i}, \ldots, \hat{x}_{j n}^{i}\right)^{\top} \in \mathbb{R}^{n}, j \in \overline{\mathcal{N}}_{i}$ are the states of the $m_{i}$ estimators $\mathcal{O}_{j}^{i}$ that are built in node $i$ with the form of

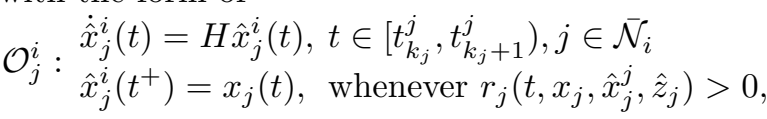

where $\overline{\mathcal{N}}_{i}=\mathcal{N}_{i} \bigcup\{i\} ; \mathcal{N}_{i}=\left\{j \in\{1,2, \ldots, N\} \mid a_{i j}>0\right\}$ is the index set of neighbors of node $i ; m_{i}$ is the cardinality of the set $\overline{\mathcal{N}}_{i}$. The increasing time sequence $\left\{t_{k_{j}}^{j}\right\}, k_{j} \in \mathbb{Z}^{+}$ when node $j$ communicates to its neighbors, is decided by the event-triggering function $r_{j}(\cdot, \cdot, \cdot, \cdot): \mathbb{R}^{+} \times \mathbb{R}^{n} \times \mathbb{R}^{n} \times$ $\mathbb{R}^{n} \rightarrow \mathbb{R}$ to be designed, i.e.,

$$
t_{k_{j}+1}^{j}=\inf \left\{t \geq t_{k_{j}}^{j} \mid r_{j}\left(t, x_{j}, \hat{x}_{j}^{j}, \hat{z}_{j}\right)>0\right\}
$$

where $t_{0}^{j}=t_{0}, \hat{z}_{j}=\sum_{l \in \mathcal{N}_{j}}\left(\hat{x}_{l}^{j}-\hat{x}_{j}^{j}\right)$. These estimators will be reinitialized as soon as node $i$ receives the sampled state from node $j$, and hence, provide node $i$ with an estimate of the current state of each of its neighbors as well as the current state of itself.

Throughout the paper, we use the following assumptions:

A1. The dynamical network (1) is connected, i.e., the outer coupling matrix $A$ is irreducible;

A2. There is no time delay for the sampling and sending executions, i.e., the time $t_{k_{i}}^{i}$ represents both the $k_{i}$ th sampling time instant and the $k_{i}$ th time when node $i$ broadcasts its sampled value $x_{i}\left(t_{k_{i}}^{i}\right)$ to its neighbors;

A3. The communication network is under an ideal circumstance, i.e., there are no time delays or data dropouts in communication.

Under Assumptions A2 and A3, all the estimators $\mathcal{O}_{i}^{j}$ for each $j \in \mathcal{N}_{i}$ will be reinitialized simultaneously using the value $x_{i}\left(t_{k_{i}}^{i}\right)$ at $t=t_{k_{i}}^{i+}$, i.e., $\hat{x}_{i}^{j}\left(t_{k_{i}}^{i+}\right)=x_{i}\left(t_{k_{i}}^{i}\right)$. This together with (4) and $t_{0}^{j}=t_{0}$ leads to

$$
\hat{x}_{i}^{j}(t)=\hat{x}_{i}^{i}(t) \quad \forall j \in \overline{\mathcal{N}}_{i}, t \geq t_{0},
$$


which implies that all the estimators $\mathcal{O}_{i}^{j}, j \in \overline{\mathcal{N}}_{i}$ that are built in different nodes to estimate the state of node $i$ have the same state response all the time. To simplify the analysis, we will not distinguish these estimators $\mathcal{O}_{i}^{j}$, and use $\hat{x}_{i}$ to replace $\hat{x}_{i}^{j}$ in the sequel. Therefore, network (1) with (4) and (3) can be simplified as

$$
\begin{aligned}
\dot{x}_{i}(t)=H x_{i}(t)+c \sum_{j=1}^{N} a_{i j} \Gamma \hat{x}_{j}(t), i=1,2, \ldots, N \\
\mathcal{O}_{i}: \\
\dot{\hat{x}}_{i}(t)=H \hat{x}_{i}(t), t \in\left[t_{k_{i}}^{i}, t_{k_{i}+1}^{i}\right) \\
\hat{x}_{i}\left(t^{+}\right)=x_{i}(t), \text { whenever } r_{i}\left(t, x_{i}, \hat{x}_{i}, \hat{z}_{i}\right)>0,
\end{aligned}
$$

where (6a) also follows by the definition of $a_{i i}$ in (2). Of course, when the communication network is not ideal, the above relationship will not hold. Then, the problem becomes more complicated, deserving more attention.

Definition 1. Let $x\left(t ; x_{0}\right)=\left(x_{1}\left(t ; x_{0}\right)^{\top}, x_{2}\left(t ; x_{0}\right)^{\top}, \ldots\right.$, $\left.x_{N}\left(t ; x_{0}\right)^{\top}\right)^{\top} \in \mathbb{R}^{n N}$ and $\hat{x}\left(t ; x_{0}\right)=\left(\hat{x}_{1}\left(t ; x_{10}\right)^{\top}, \hat{x}_{2}\left(t ; x_{20}\right)^{\top}\right.$ $\left.\ldots, \hat{x}_{N}\left(t ; x_{N 0}\right)^{\top}\right)^{\top} \in \mathbb{R}^{n N}$ be a solution to network (6) with the initial condition $x_{0}=\left(x_{10}^{\top}, x_{20}^{\top}, \ldots, x_{N 0}^{\top}\right)^{\top}$ and $x_{i 0}=x_{i}\left(t_{0}\right)$. Then we say network (6) achieves synchronization asymptotically, if $x\left(t ; x_{0}\right)$ and $\hat{x}\left(t ; x_{0}\right)$ exist for every initial condition $x_{0} \in \mathbb{R}^{n N}$ and $t>t_{0}$, and further if

$$
\lim _{t \rightarrow \infty}\left|x\left(t ; x_{0}\right)\right|_{\mathcal{A}_{s}}=0,
$$

where $\mathcal{A}_{s}=\left\{x \in \mathbb{R}^{n N} \mid x_{1}=x_{2}=\cdots=x_{N}\right\}$ is called the synchronization manifold of network (6), and $|x|_{\mathcal{A}_{s}}$ is the Euclidean point-to-set distance, namely

$$
|x|_{\mathcal{A}_{s}}=d\left(x, \mathcal{A}_{s}\right)=\inf _{y \in \mathcal{A}_{s}}\|x-y\| .
$$

\section{SYNCHRONIZATION BY DISTRIBUTED EVENT-TRIGGERED CONTROL}

In order to achieve the main goal of the paper, we define the error vector $e_{i}(t)=\hat{x}_{i}(t)-x_{i}(t)$, and denote $x=$ $\left(x_{1}^{\top}, x_{2}^{\top}, \ldots, x_{N}^{\top}\right)^{\top}$ as well as $e=\left(e_{1}^{\top}, e_{2}^{\top}, \ldots, e_{N}^{\top}\right)^{\top}$. Then, network model (6a) can be rewritten as follows

$$
\dot{x}=\left(I_{N} \otimes H+c A \otimes \Gamma\right) x+(c A \otimes \Gamma) e .
$$

Since the matrix $A$ is irreducible, symmetric, and has zero row sums (2), there always exists a unitary matrix $\Psi=$ $\left(\psi_{1}, \psi_{2}, \ldots, \psi_{N}\right) \in \mathbb{R}^{N \times N}$ with $\psi_{i}=\left(\psi_{i 1}, \psi_{i 2}, \ldots, \psi_{i N}\right)^{\top} \in$ $\mathbb{R}^{N}$ and $\Psi^{\top} \Psi=I_{N}$ such that

$$
\Psi^{\top} A \Psi=\Lambda=\operatorname{diag}\left(\lambda_{1}, \lambda_{2}, \ldots, \lambda_{N}\right),
$$

where $0=\lambda_{1}>\lambda_{2} \geq \lambda_{3} \geq \cdots \geq \lambda_{N}$, and $\lambda_{1}=0$ with algebraic multiplicity one. Furthermore, we can choose $\psi_{1}=\frac{1}{\sqrt{N}}(1,1, \ldots, 1)^{\top}$ for $\lambda_{1}=0$, and this leads to

$$
\sum_{j=1}^{N} \psi_{i j}=0 \text { for all } i=2,3, \ldots, N \text {. }
$$

Let $\Phi=\left(\psi_{2}, \psi_{3}, \ldots, \psi_{N}\right) \in \mathbb{R}^{N \times(N-1)}, \Lambda_{1}=\Phi^{\top} A \Phi=$ $\operatorname{diag}\left\{\lambda_{2}, \lambda_{3}, \ldots, \lambda_{N}\right\}$ and $\bar{\Phi}=\Phi \otimes I_{n}$. Defining $y=\bar{\Phi}^{\top} x$, we have

$$
\begin{aligned}
\dot{y}(t)= & \bar{\Phi}^{\top}\left(\left(I_{N} \otimes H\right) x+(c A \otimes \Gamma)\left(I_{N n}-\bar{\Phi} \bar{\Phi}^{\top}\right.\right. \\
& \left.\left.+\bar{\Phi} \bar{\Phi}^{\top}\right)(x+e)\right) \\
= & \left(I_{N-1} \otimes H+c \Lambda_{1} \otimes \Gamma\right) y+\left(c \Lambda_{1} \otimes \Gamma\right) \bar{\Phi}^{\top} e,
\end{aligned}
$$

where we use the properties $\bar{\Phi}^{\top}\left(I_{N} \otimes H\right)=\left(I_{N-1} \otimes H\right) \bar{\Phi}^{\top}$ and $(c A \otimes \Gamma)\left(I_{N n}-\bar{\Phi} \bar{\Phi}^{\top}\right)=0$, which are supported by the fact $\Phi^{\top} \Phi=I_{N-1}, A 1_{N}=0, I_{N}-\Phi \Phi^{\top}=I_{N}-$ $U=\frac{1}{N} 1_{N \times N}$ and $U=\Phi \Phi^{\top}=I_{N}-\frac{1}{N} 1_{N \times N}$.

Let $H_{i}=H+\Gamma_{i}$ with $\Gamma_{i}=c \lambda_{i} \Gamma, i=2,3, \ldots, N$, and denote $\bar{H}=I_{N-1} \otimes H+c \Lambda_{1} \otimes \Gamma=\operatorname{diag}\left\{H_{2}, H_{3}, \ldots, H_{N}\right\}$ and $\bar{\Lambda}=c \Lambda_{1} \otimes \Gamma=\operatorname{diag}\left\{\Gamma_{2}, \Gamma_{3}, \ldots, \Gamma_{N}\right\}$. Then, system (10) can be simplified as

$$
\dot{y}=\bar{H} y+\bar{\Lambda}\left(\bar{\Phi}^{\top} e\right) .
$$

It is shown in Liu et al. (2012) that $\|y\|=\left\|\bar{\Phi}^{\top} x\right\|=|x|_{\mathcal{A}_{s}}$. Therefore, if $\lim _{t \rightarrow \infty}\|y(t)\|=0$, then by the definition of synchronization, one can conclude that network (6) achieves synchronization asymptotically. We summarize this result in the following lemma.

Lemma 1. If system (11) and (6b) has the property

$$
\lim _{t \rightarrow \infty}\|y(t)\|=0
$$

then network (6) achieves synchronization asymptotically.

Now, we will discuss the synchronization problem of (6) by designing a proper event-triggering rule. In the case where all states of network (6) can be accessed for the design purpose, we can get a centralized event-triggering rule.

Theorem 2. If there exist positive definite matrices $P_{i} \in$ $\mathbb{R}^{n \times n}$ such that

$$
H_{i}^{\top} P_{i}+P_{i} H_{i}=-2 I_{n}, i=2,3, \ldots, N,
$$

then network (6) is globally asymptotically synchronized under the sampling time sequence determined by the centralized event-triggering function $r(e, x)=\left\|\bar{\Phi}^{\top} e\right\|-$ $\frac{\delta}{\alpha}\left\|\bar{\Phi}^{\top} x\right\|$, i.e.,

$$
t_{k+1}=\inf \left\{t>t_{k} \mid r(e, x)>0\right\}
$$

where $\delta \in(0,1), \alpha=\max _{i=2,3, \ldots, N}\left\{-c \lambda_{i}\left\|P_{i} \Gamma\right\|\right\}$. Moreover, no Zeno behavior occurs in (6) for all $t \geq t_{0}$.

Proof. First, we claim that with (13), there exists a $\tau^{*}>0$ such that $\tau_{k}=t_{k+1}-t_{k} \geq \tau^{*}, \forall k \in \mathbb{Z}$. To prove this, we use the method proposed in Tabuada (2007) by calculating the derivative of $\left\|\bar{\Phi}^{\top} e\right\| /\left\|\bar{\Phi}^{\top} x\right\|$, i.e.,

$$
\begin{aligned}
\frac{d}{d t} \frac{\left\|\bar{\Phi}^{\top} e\right\|}{\left\|\bar{\Phi}^{\top} x\right\|} & =\frac{\left(\bar{\Phi}^{\top} e\right)^{\top}\left(\bar{\Phi}^{\top} \dot{e}\right)\|y\|}{\left\|\bar{\Phi}^{\top} e\right\|\|y\|^{2}}-\frac{\left\|\bar{\Phi}^{\top} e\right\| y^{\top} \dot{y}}{\|y\|\|y\|^{2}} \\
& \leq \frac{\left\|\bar{\Phi}^{\top} \dot{e}\right\|}{\|y\|}+\frac{\left\|\bar{\Phi}^{\top} e\right\|\|\dot{y}\|}{\|y\|^{2}} .
\end{aligned}
$$

By the definition of $e_{i}, i=1,2, \ldots, N$, and with the same procedure used in (9) and (10), we have

$$
\bar{\Phi}^{\top} \dot{e}=\left(I_{N-1} \otimes H-\bar{\Lambda}\right) \bar{\Phi}^{\top} e-\bar{\Lambda} y .
$$

Thus, we can get an upper bound on $\left\|\bar{\Phi}^{\top} \dot{e}\right\|$, i.e.,

$$
\left\|\bar{\Phi}^{\top} \dot{e}\right\| \leq\left(\left\|I_{N-1} \otimes H\right\|+\|\bar{\Lambda}\|\right)\left\|\bar{\Phi}^{\top} e\right\|+\|\bar{\Lambda}\|\|y\| .
$$

Also, we can get an upper bound on $\|\dot{y}\|$ from (11), i.e.,

$$
\|\dot{y}\| \leq\left(\left\|I_{N-1} \otimes H\right\|+\|\bar{\Lambda}\|\right)\|y\|+\|\bar{\Lambda}\|\left\|\bar{\Phi}^{\top} e\right\| .
$$

Combining (14) with (16) and (17) leads to

$$
\frac{d}{d t} \frac{\left\|\bar{\Phi}^{\top} e\right\|}{\left\|\bar{\Phi}^{\top} x\right\|} \leq a \frac{\left\|\bar{\Phi}^{\top} e\right\|^{2}}{\|y\|^{2}}+2(a+b) \frac{\left\|\bar{\Phi}^{\top} e\right\|}{\|y\|}+a,
$$

where $a=\|\bar{\Lambda}\|$ and $b=\left\|I_{N-1} \otimes H\right\|$. Consider the differential equation:

$$
\dot{\phi}=a \phi^{2}+2(a+b) \phi+a,
$$

then one can conclude that $\left\|\bar{\Phi}^{\top} e\right\| /\left\|\bar{\Phi}^{\top} x\right\| \leq \phi\left(t, \phi\left(t_{0}\right)\right)$ whenever $\left\|\bar{\Phi}^{\top} e\left(t_{0}\right)\right\| /\left\|\bar{\Phi}^{\top} x\left(t_{0}\right)\right\| \leq \phi_{0}$, where $\phi\left(t, \phi\left(t_{0}\right)\right)$ is 
the solution of (19) with initial condition $\phi\left(t_{0}, \phi\left(t_{0}\right)\right)=$ $\phi\left(t_{0}\right)$. Therefore, the inter-execution intervals $\tau_{k}$ can be lower bounded by the time during which $\phi$ evolves from 0 to $\frac{\delta}{\alpha}$, i.e., $\phi\left(\tau^{*}, 0\right)=\frac{\delta}{\alpha}$. Such a $\tau^{*}$ can be obtained by solving the differential equation (19), namely

$$
\tau^{*}=\frac{1}{2 a c} \ln \left|\frac{\delta+\alpha c_{1}-\alpha c}{\delta+\alpha c_{1}+\alpha c}\right|+\frac{c_{0}}{a}>0
$$

with $c_{1}=\frac{a+b}{a}, c=\sqrt{\frac{(a+b)^{2}-a^{2}}{a}}>0$ and $c_{0}=$ $-\frac{1}{2 c} \ln \left|\frac{c_{1}-c}{c_{1}+c}\right|$. Thus, no Zeno behavior will occur in network (6) under event-triggering rule (13) for all $t \geq t_{0}$.

Now, select the following Lyapunov function candidate

$$
V=y^{\top} P y,
$$

where $P=\operatorname{diag}\left\{P_{2}, P_{3}, \ldots, P_{N}\right\}$ and $P_{i}, i=2,3, \ldots, N$ are positive definite matrix solutions of (12). Then along the trajectories of system (11), one has

$$
\dot{V}=\dot{y}^{\top} P y+y^{\top} P \dot{y} \leq-2\|y\|^{2}-2\|P \bar{\Gamma}\|\left\|\bar{\Phi}^{\top} e\right\| .
$$

The event-triggering rule (13) ensures that $\left\|\bar{\Phi}^{\top} e\right\| \leq$ $\frac{\delta}{\alpha}\left\|\bar{\Phi}^{\top} x\right\|=\frac{\delta}{\alpha}\|y\|$ for all $t \geq t_{0}$. This together with $(22)$ makes the following inequality hold

$$
\dot{V} \leq-2(1-\delta)\|y\|^{2} \text {. }
$$

Therefore, the equilibrium point $y=0$ of system (11) is asymptotically stable, i.e., $\lim _{t \rightarrow \infty}\|y(t)\|=0$. Applying Lemma 1 proves the result.

Remark 1. For a dynamical network whose nodes interact with their neighbors all the time, condition (12) is a necessary and sufficient condition for asymptotic synchronization (Trentelman et al. (2013)). Hence, for a network with event-based communication (6a), it is reasonable to require that (12) holds in Theorem 2.

Remark 2. In practice, a centralized event-triggering rule (13) is usually hard to implement. For one thing, it may be costly and time consuming to gather the global information for the design purpose. For another, each node normally can only get limited information from its neighbors - the sampled state value of its neighbors, which can only be received at certain discrete time instants, rather than $x(t)$ for all $t>t_{0}$. Therefore, a distributed event-triggering rule for a given node which only relies on the information that the node can get is desirable.

In order to design such a distributed event-triggering rule for each node $i$ by only using the information of $x_{i}(t)$, $\hat{x}_{i}(t)$, and $\hat{x}_{j}(t), j \in \mathcal{N}_{i}$, we first give a lemma which will be used in the proof the main result. For the proof of the lemma, please refer to Zhao et al. (2012).

Lemma 3. (Zhao et al. (2012)). Consider the time-varying nonlinear system

$$
\dot{x}=f(x, t),
$$

where $f: \mathbb{R}^{n} \times \mathbb{R}^{+} \rightarrow \mathbb{R}^{n}$ is continuous. Suppose that for any $M>0$, there exists $\eta=\eta_{M}$, which may depend on $M$, such that

$$
\|f(x, t)\| \leq \eta_{M}, \forall t \geq 0 \text { and }\|x\|<M .
$$

Then, if there exist a nonnegative bounded function $g(t)$ defined on $\mathbb{R}^{+}$, a smooth function $V(x, t): \mathbb{R}^{n} \times \mathbb{R}^{+} \rightarrow \mathbb{R}$ and $\mathcal{K}$ functions $\alpha_{1}, \alpha_{2}$ and $\alpha_{3}$ satisfying

$$
\alpha_{1}(\|x\|) \leq V(x, t) \leq \alpha_{2}(\|x\|),
$$

$$
\begin{aligned}
\dot{V}(x, t) & \triangleq\left(\frac{\partial}{\partial x} V(x, t)\right)^{\top} f(x, t)+\frac{\partial}{\partial t} V(x, t) \\
& \leq-\alpha_{3}(\|x\|), \text { whenever }\|x\| \geq g(t),
\end{aligned}
$$

and $V(s, t)$ and $\dot{V}(x, t)$, as functions of $x$, are equicontinuous on any bounded region of $\mathbb{R}^{n}$, uniformly with respect to $t$, the solution $x(t)$ of system (24) converges to the set

$$
\mathcal{Q}=\bigcap_{T \geq b>0}\left(\bigcup_{a \geq T} \mathcal{Q}_{a, b}\right)
$$

where

$$
\begin{gathered}
\mathcal{Q}_{a, b}=\left\{x \mid V(x, a) \leq \sup _{y \in \Omega, s \geq b}\{V(y, s)\}\right\}, \\
\Omega=\left\{x \in \mathbb{R}^{n} \mid\|x\| \leq \overline{\lim }_{t \rightarrow \infty} g(t)\right\},
\end{gathered}
$$

and $\varlimsup_{t \rightarrow \infty} g(t)$ is the upper limit of $g(t)$. Moreover, if $\varlimsup_{t \rightarrow \infty} g(t)=0$, then system (24) is asymptotically stable.

Theorem 4. If there exist positive definite matrix solutions $P_{i} \in \mathbb{R}^{n \times n}$ to the Lyapunov equations in (12), then network (6) can achieve synchronization asymptotically under the distributed event-triggering rule

$$
t_{k_{i}+1}^{i}=\inf \left\{t \geq t_{k_{i}}^{i} \mid r_{i}\left(t, e_{i}, \hat{z}_{i}\right)>0\right\},
$$

where $r_{i}\left(t, e_{i}, \hat{z}_{i}\right)=\left\|e_{i}\right\|-\rho \sqrt{\left\|\hat{z}_{i}\right\|^{2}+\mathrm{e}^{-2 \gamma t}} ; \rho=\frac{-\delta}{\lambda_{N}(\alpha+\delta)}>$ $0 ; \lambda_{N}<0$ is the smallest eigenvalue of $A ; \gamma$ is a positive constant such that $\gamma<\lambda_{\min }$ with $\lambda_{\min }=$ $-\max _{i \in\{2,3, \ldots, N\}}\left\{\operatorname{Re}\left(\lambda\left(H_{i}\right)\right)\right\} ; \alpha$ and $\delta$ are defined the same as in Theorem 2. Moreover, no Zeno behavior occurs in (6) for all $t \geq t_{0}$.

Proof. We divide the proof into two steps: 1). to show the existence of a lower bound on the inter-execution intervals for event-triggering rule (31), and 2). to prove the asymptotic convergency of network (6) to $\mathcal{A}_{s}$.

1). Instead of discussing event-triggering rule (31), we consider the following event-triggering rule

$$
t_{k+1}^{i}=\inf \left\{t \geq t_{k}^{i} \mid\left\|e_{i}\right\|>\rho \mathrm{e}^{-\gamma t}\right\} .
$$

By solving differential equation (11) and using the method proposed in Guinaldo et al. (2011), we can get an upper bound on $\|y\|$, i.e.,

$$
\begin{aligned}
\|y\| \leq & \left\|\mathrm{e}^{\bar{H}\left(t-t_{0}\right)}\right\|\left\|y\left(t_{0}\right)\right\|+\int_{t_{0}}^{t}\left\|\mathrm{e}^{\bar{H}(t-\theta)}\right\|\|\bar{\Lambda}\|\left\|\bar{\Phi}^{\top} e(\theta)\right\| \mathrm{d} \theta \\
\leq & k_{0} \mathrm{e}^{-\lambda_{\min }\left(t-t_{0}\right)}\left\|y\left(t_{0}\right)\right\| \\
& +k_{0} \int_{t_{0}}^{t} \mathrm{e}^{-\lambda_{\min }(t-\theta)}\|\bar{\Lambda}\|\left\|\bar{\Phi}^{\top} e(\theta)\right\| \mathrm{d} \theta
\end{aligned}
$$

where $k_{0}=\left\|U_{T}\right\|\left\|U_{T}{ }^{-1}\right\|$, and $U_{T}$ is a nonsingular matrix such that $U_{T} \bar{H} U_{T}{ }^{-1}=D$ with $D$ being the diagonal matrix composed of the eigenvalues of $\bar{H}$. The eventtriggering rule (32) guarantees that $\left\|e_{i}\right\| \leq \rho \mathrm{e}^{-\gamma t}$ for all $t \geq t_{0}$, which together with the property $\|\bar{\Phi}\|=1$ gives

$$
\left\|\bar{\Phi}^{\top} e\right\| \leq\|\bar{\Phi}\|\|e\|=\|e\|<\sqrt{N} \rho \mathrm{e}^{-\gamma t} .
$$

Putting the above inequality into (33) gives

$$
\|y\| \leq k_{0}\left\|y\left(t_{0}\right)\right\| \mathrm{e}^{\lambda_{\min } t_{0}} \mathrm{e}^{-\lambda_{\min } t}+\frac{k_{0} \rho \sqrt{N}\|\bar{\Lambda}\|}{\lambda_{\min }-\alpha} \mathrm{e}^{-\gamma t} .
$$

In addition, the dynamics of $e_{i}$ can be rewritten as

$$
\dot{e}_{i}=H e_{i}-\Gamma \hat{z}_{i}
$$


which gives

$$
\begin{aligned}
\left\|\dot{e}_{i}\right\| & \leq\|H\|\left\|e_{i}\right\|+\|\Gamma\|\left\|\hat{z}_{i}\right\| \\
& \leq \rho\|H\| \mathrm{e}^{-\gamma t}+\|\Gamma\|\left(\left\|\left(A \otimes I_{n}\right) x\right\|+\left\|\left(A \otimes I_{n}\right) e\right\|\right) \\
& \leq \rho\left(\|H\|-\lambda_{N} \sqrt{N}\|\Gamma\| \mathrm{e}^{-\gamma t}+\|\Gamma\|\|z\|,\right.
\end{aligned}
$$

where $z=\left(A \otimes I_{n}\right) x$, and $\left\|A \otimes I_{n}\right\|=-\lambda_{N}$. Moreover,

$$
\begin{aligned}
\|z\|^{2} & =x^{\top}\left(A^{2} \otimes I_{n}\right) x \leq \lambda_{N}^{2} x^{\top}\left(U^{2} \otimes I_{n}\right) x \\
& =\lambda_{N}^{2}\left\|\bar{\Phi}^{\top} x\right\|^{2}=\lambda_{N}^{2}\|y\|^{2},
\end{aligned}
$$

which comes from $A^{2} \leq \lambda_{N}^{2} U^{2}$ and $U^{2}=U=\bar{\Phi} \bar{\Phi}^{\top}$. Therefore, the inequality (36) becomes

$$
\left\|\dot{e}_{i}\right\| \leq \rho\left(\|H\|-\lambda_{N} \sqrt{N}\|\Gamma\|\right) \mathrm{e}^{-\gamma t}-\lambda_{N}\|\Gamma\|\|y\| .
$$

From (38) and (34), for all $t \in\left[t_{k_{i}}^{i}, t_{k_{i}+1}^{i}\right)$ and $k \in \mathbb{Z}$, we have

$$
\left\|\dot{e}_{i}\right\| \leq k_{1} \mathrm{e}^{-\lambda_{\min } t}+k_{2} \mathrm{e}^{-\gamma t} \leq k_{1} \mathrm{e}^{-\lambda_{\min } t_{k_{i}}^{i}}+k_{2} \mathrm{e}^{-\gamma t_{k_{i}}^{i}},
$$

where $k_{1}=k_{0}\|\Gamma\|\left\|y\left(t_{0}\right)\right\| \mathrm{e}^{-\lambda_{\min } t_{0}}$ and $k_{2}=\frac{k_{0} \rho \sqrt{N}\|\bar{\Lambda}\|}{\lambda_{\min }-\gamma}+$ $\rho\left(\|H\|-\lambda_{N} \sqrt{N}\|\Gamma\|\right)$. Thus, we get

$$
\begin{aligned}
\left\|e_{i}(t)\right\| & =\left\|\int_{t_{k_{i}}^{i}}^{t} \dot{e}_{i}(\theta) \mathrm{d} \theta\right\| \leq \int_{t_{k_{i}}^{i}}^{t}\left\|\dot{e}_{i}(\theta)\right\| \mathrm{d} \theta \\
& \leq\left(k_{1} \mathrm{e}^{-\lambda_{\min } t_{k_{i}}^{i}}+k_{2} \mathrm{e}^{-\gamma t_{k_{i}}^{i}}\right)\left(t-t_{k_{i}}^{i}\right) \\
& \leq \mathrm{e}^{-\gamma t_{k_{i}}^{i}}\left(k_{1}+k_{2}\right) \tau_{k_{i}},
\end{aligned}
$$

where $\tau_{k_{i}}=t-t_{k_{i}}^{i}$, and the second inequality follows from $\lambda_{\min }>\gamma>0$. Because at $t=t_{k_{i}}^{i}, e(t)=0$ and according to (31), the next event will not be triggered until $\left\|e_{i}(t)\right\|=\rho \mathrm{e}^{-\gamma t}$. Therefore, the inter-execution intervals $\tau_{k_{i}}^{*} \leq t_{k_{i}+1}^{i}-t_{k_{i}}^{i}$ can be lower bounded by the solution $\tau_{i}^{*}$ of the equation $\left(k_{1}+k_{2}\right) \tau_{i}^{*}=\rho \mathrm{e}^{-\gamma \tau_{i}^{*}}$ which is strictly positive. Please refer to Seyboth et al. (2013) for details.

Apparently, the inter-execution intervals of event-triggering rule (31) are greater than those of rule (32) because $\sqrt{\left\|\hat{z}_{i}\right\|^{2}+\mathrm{e}^{-2 \gamma t}} \geq \mathrm{e}^{-\gamma t}$ with $\left\|\hat{z}_{i}\right\| \geq 0$, and thus there also exists a positive lower bound on the inter-execution intervals for (31).

2 ). The event-triggering rule (31) guarantees that

$$
\left\|e_{i}\right\|<\rho \sqrt{\left\|\hat{z}_{i}\right\|^{2}+\mathrm{e}^{-2 \gamma t}}, \quad \forall t \geq t_{0} .
$$

Since

$\sum_{i=1}^{N}\left(\left\|\hat{z}_{i}\right\|^{2}+\mathrm{e}^{-2 \gamma t}\right)=\|\hat{z}\|^{2}+N \mathrm{e}^{-2 \gamma t} \leq\left(\|\hat{z}\|+\sqrt{N} \mathrm{e}^{-\gamma t}\right)^{2}$,

we have

$$
\begin{aligned}
\|e\| & <\rho\left(\|\hat{z}\|+\sqrt{N} \mathrm{e}^{-\gamma t}\right) \\
& =\rho\left(\left\|\left(A \otimes I_{n}\right)(x+e)\right\|+\sqrt{N} \mathrm{e}^{-\gamma t}\right) \\
& \leq \rho\left(\|z\|-\lambda_{N}\|e\|+\sqrt{N} \mathrm{e}^{-\gamma t}\right) .
\end{aligned}
$$

As $\rho=\frac{-\delta}{\lambda_{N}(\alpha+\delta)}$, inequality (41) is equivalent to

$$
\|e\|<\frac{-\delta}{\alpha \lambda_{N}}\left(\|z\|+\sqrt{N} \mathrm{e}^{-\gamma t}\right) .
$$

Using (37) again leads to

$$
\|e\|<\frac{\delta}{\alpha}\left(\left\|\bar{\Phi}^{\top} x\right\|-\frac{\sqrt{N} \mathrm{e}^{-\gamma t}}{\lambda_{N}}\right) .
$$

Combining $\left\|\bar{\Phi}^{\top} e\right\| \leq\|e\|$ with (42) gives

$$
\left\|\bar{\Phi}^{\top} e\right\|<\frac{\delta}{\alpha}\left(\left\|\bar{\Phi}^{\top} x\right\|-\frac{\sqrt{N} \mathrm{e}^{-\gamma t}}{\lambda_{N}}\right) .
$$

Now, select the Lyapunov candidate $V=y^{\top} P y$ with $P=$ $\operatorname{diag}\left\{P_{2}, P_{3}, \ldots, P_{N}\right\}$, then with (44) and $\left\|\bar{\Phi}^{\top} x\right\|=\|y\|$, the derivative of $V$ along system (11) satisfies

$$
\begin{aligned}
\dot{V} & \leq-2\|y\|^{2}+2 \alpha\|y\|\left\|\bar{\Phi}^{\top} e\right\| \\
& \leq-2(1-\delta)\|y\|^{2}+2 \rho_{1}\|y\| \mathrm{e}^{-\gamma t} \\
& =-2\left(1-\delta-\delta_{1}\right)\|y\|^{2}-2\|y\|\left(\delta_{1}\|y\|-\rho_{1} \mathrm{e}^{-\gamma t}\right) \\
& \leq-2\left(1-\delta-\delta_{1}\right)\|y\|^{2}, \text { when }\|y\| \geq \rho_{2} \mathrm{e}^{-\gamma t},
\end{aligned}
$$

where $0<\delta_{1}<1-\delta, \rho_{1}=-\frac{\delta \sqrt{N}}{\frac{\lambda_{N}}{N_{L}}}>0, \rho_{2}=-\frac{\delta \sqrt{N}}{\delta_{1} \lambda_{N}}>0$. Let $g(t)=\rho_{2} \mathrm{e}^{-\gamma t}$, so we have $\lim _{t \rightarrow \infty} g(t)=0$. Applying Lemma 3 , we get that the equilibrium point $y=0$ of system (11) is asymptotically stable, and thus the network can achieve synchronization asymptotically.

Remark 3. Asymptotic synchronization was also investigated for a similar dynamical network by using selftriggered control in De Persis (2013). The main differences between the results proposed in De Persis (2013) and ours are twofold. First, in De Persis (2013), each node will require the updated information from its neighbors when the self-triggering condition is fulfilled, and therefore, bidirectional communication is needed; whereas in our paper, a node will broadcast its sampled state to its neighbors directly as long as its own event-triggering condition is satisfied, and hence, only undirectional communication is required. Second, the system matrix of the node dynamics is required to have purely imaginary eigenvalues with unitary geometric multiplicity in De Persis (2013), and our results don't rely on such a restriction.

\section{AN EXAMPLE}

This section gives an example to show the effectiveness of the proposed results. Here, we adopt the example used in Liu et al. (2012), where the network consists of 10 nodes. The parameters of the network are as follows: $c=1$,

$$
\begin{gathered}
H=\left(\begin{array}{cc}
0 & -0.5 \\
0.5 & 0
\end{array}\right), \Gamma=\left(\begin{array}{cccccc}
0.25 & 0 \\
-1 & 0.25
\end{array}\right), \\
A=\left(\begin{array}{cccccccccc}
-4 & 1 & 1 & 0 & 0 & 0 & 0 & 0 & 1 & 1 \\
1 & -4 & 1 & 1 & 0 & 0 & 0 & 0 & 0 & 1 \\
1 & 1 & -4 & 1 & 1 & 0 & 0 & 0 & 0 & 0 \\
0 & 1 & 1 & -4 & 1 & 1 & 0 & 0 & 0 & 0 \\
0 & 0 & 1 & 1 & -4 & 1 & 1 & 0 & 0 & 0 \\
0 & 0 & 0 & 1 & 1 & -4 & 1 & 1 & 0 & 0 \\
0 & 0 & 0 & 0 & 1 & 1 & -4 & 1 & 1 & 0 \\
0 & 0 & 0 & 0 & 0 & 1 & 1 & -4 & 1 & 1 \\
1 & 0 & 0 & 0 & 0 & 0 & 1 & 1 & -4 & 1 \\
1 & 1 & 0 & 0 & 0 & 0 & 0 & 1 & 1 & -4
\end{array}\right) .
\end{gathered}
$$

Since $H$ has two eigenvalues $\pm 0.5 i$ on the imaginary axis, the network cannot asymptotically synchronize to its equilibrium point, but to a stable time-varying solution.

When all the information of network (6) is available for the design purpose, then we can design centralized eventtriggering rule (13) to synchronize the network. Figure 1 gives the simulation results under the designed centralized event-triggering rule with $\delta=0.9$ and $\alpha=2.9061$, which shows that the network achieves synchronization asymptotically, and the minimum inter-execution interval is $\tau^{*}=0.0186 \mathrm{~s}$ for $t$ from $0 \mathrm{~s}$ to $25 \mathrm{~s}$. 

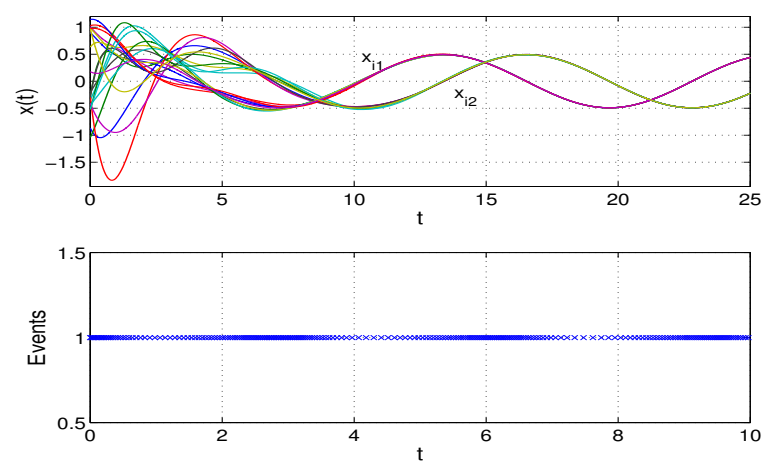

Fig. 1. Simulation for centralized event-triggering rule.

For the network where each node can only get limited information, we can design a distributed event-triggering rule (31) to achieve asymptotic synchronization based on Theorem 4. Figure 2 shows the state of the entire network and sampling times of each node, where $\delta=0.9$, $\alpha=2.9061, \lambda_{N}=-6.1518, \lambda_{\min }=0.2972$, and $\gamma=0.29$. The minimum inter-execution interval $\tau_{i}^{*}$ for each node for $t$ from 0 s to $25 \mathrm{~s}$ is given in Table 1 .
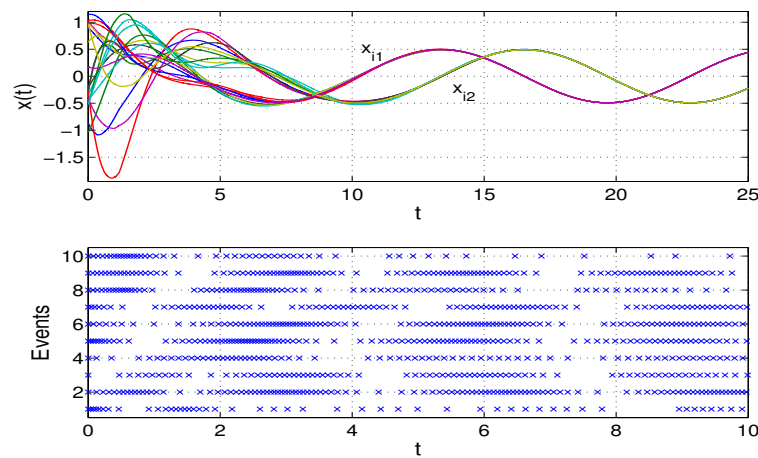

Fig. 2. Simulation for the distributed event-triggering rule.

Table 1. The minimum inter-execution interval

\begin{tabular}{cccccc}
\hline \hline & Node 1 & Node 2 & Node 3 & Node 4 & Node 5 \\
\hline$\tau_{i}^{*}$ & 0.0386 & 0.0531 & 0.0691 & 0.0665 & 0.0378 \\
\hline \hline & Node 6 & Node 7 & Node 8 & Node 9 & Node 10 \\
\hline$\tau_{i}^{*}$ & 0.0501 & 0.0469 & 0.0452 & 0.0507 & 0.0480 \\
\hline
\end{tabular}

\section{CONCLUSION}

This paper has investigated the asymptotic synchronization problem of a dynamical network by using the distributed event-triggered control method. With the help of the introduced estimators, a distributed event-triggering rule for each node has been explored, which only relies on the state of the node and the states of the introduced estimators. It has been shown that the network can achieve synchronization asymptotically with the proposed eventtriggering rule, and no Zeno behavior occurs during the whole evolution of the system. It is worth pointing out that quantization effect, time-delay and data packet dropout are common phenomena which will definitely affect the synchronization of a network with event-based communication. Thus, it appears that the synchronization of such networks with imperfect communication is an important issue to pursue further for both theoretical interest and practical consideration.

\section{REFERENCES}

De Persis, C. (2013). On self-triggered synchronization of linear systems. In IFAC NecSys2013, accepted. Koblenz, Germany

De Persis, C., Sailer, R., and Wirth, F. (2011). On a smallgain approach to distributed event-triggered control. In IFAC World Congress, 2401-2406. Milan, Italy.

Demir, O. and Lunze, J. (2012). Event-based synchronziation of multi-agent systems. In the 4th IFAC Conf. on Analysis and Design of Hybrid Systems, 1-6. Eindhoven, The Netherlands.

Dimarogonas, D.V. (2011). $L_{2}$ gain stability analysis of event-triggered agreement protocols. In IEEE Conf. Decision and Control, 2130-2135. Orlando, USA.

Dimarogonas, D.V. and Johansson, K.H. (2009). Eventbased control for multi-agent systems. In IEEE Conf. Decision and Control, 7131-7136. Shanghai, China.

Donkers, M.C.F. and Heemels, W.P.M.H. (2012). Outputbased event-triggered control with guaranteed $L_{\infty}$-gain and improved and decentralised event-triggering. IEEE Trans. Automatic Control, 57(6), 1362-1376.

Garcia, E. and Antsaklis, P.J. (2013). Model-based eventtriggered control for systems with quantization and time-varying network delays. IEEE Trans. Automatic Control, 58(2), 422-434.

Guinaldo, M., Dimarogonas, D.V., Johansson, K.H., Sánchez, J., and Dormido, S. (2011). Distributed eventbased control for interconnected linear systems. In IEEE Conf. on Decision and Control and European Control Conf., 2553-2558. Orlando, USA.

Heemels, W.P.M.H., Johansson, K.H., and Tabuada, P. (2012). An introduction to event-triggered and selftriggered control. In IEEE Conf. Decision and Control, 3270-3285. Maui, USA.

Liu, T., Hill, D.J., and Liu, B. (2012). Synchronization of dynamical networks with distributed event-based communication. In IEEE Conf. Decision and Control, 7199-7204. Maui, USA.

Mazo Jr., M. and Cao, M. (2011). Decentralized eventtriggered control with asynchronous updates. In IEEE Conf. Decision and Control, 2547-2552. Orlando, USA. Seyboth, G.S., Dimarogonas, D.V., and Johansson, K.H. (2013). Event-based broadcasting for multi-agent average consensus. Automatica, 49, 245-252.

Tabuada, P. (2007). Event-triggered real-time scheduling of stabilizing control tasks. IEEE Trans. Automatic Control, 52(9), 1680-1685.

Trentelman, H.L., Takaba, K., and Monshizadeh, N. (2013). Robust synchronization of uncertain linear multi-agent systems. IEEE Trans. Automatic Control, $58(6), 1511-1523$.

Wang, X.F. and Lemmon, M.D. (2011). Event-triggering in distributed networked control systems. IEEE Trans. Automatic Control, 56(3), 586-601.

Zhao, J., Hill, D.J., and Liu, T. (2012). Global bounded synchronization of general dynamical networks with non-identical nodes. IEEE Trans. Automatic Control, 57(10), 2656-2662. 